\title{
Behcet disease: a case study
}

INTRODUCTION: Behçet disease ( BD) is more common in Mediterranean countries and the Middle East, and in Japan the prevalence is 10 per 100,000 of the population (Chamberlaine, 1977, 1978). The etiology of the disease is unknown, but an immunological basis seems possible. The histology is of vasculitis, particularly involving venules and capillaries, with mononuclear cell infiltration. Circulating autoantibodies to oral mucosa were first described in 1963 (Oshima et al., 1963). The main clinical features of Behget's disease are relapsing iridocyclitis with recurrent oral and genital ulceration.
METHODS: We report a cross-sectional and multicentric study ( in 3 centers in Algeria) in a series of 70 cases followed in rheumatology since 1986 to date. The diagnosis of BD was made on international criteria (ICBD).

Table 1: Clinical data collected in the Behcets's cohort.

\section{RESULTS:}

70 cases reported: 51 male for 19 female, index ratio $=3$, the average age of diagnosis is 37 years (18-72), the juvenile onset seen in 5 cases. Mucocutaneous disorders are reported as buccal aphtosis in 38\% (bipolar aphtosis $62 \%)$, pseudofolliculitis in $55 \%$, erythema nodosum in $16 \%$, vasculitis in $9 \%$, positif pathergy test in $10 \%$, joint disorders reported in $95 \%$, thrombophlebitis in $11 \%$ and ocular involvement in 39\%, neurologic damage in $18 \%$ ( Table 1), inflammatory syndrome seen in 49\%, HLA B5 in $23 \%$.

Gastrointestinal involvement was more frequent than in patients without vascular involvement $(p<0.001)$; ocular involvement was less frequent $(p<0.05)$. Only 3 patients $(12 \%)$ required surgery. Patients received prednisone and immunosuppressants, including etanercept, for vascular and/or concurrent gastrointestinal involvement. Nine patients received warfarin, without bleeding complications.Two patient died during the observation period.

\begin{tabular}{ll|}
\hline Feature & Number (\%) \\
\hline Buccal aphtosis & $27(37.8)$ \\
\hline Bipolar aphtosis & $6(9.1)$ \\
\hline Pseudofolliculitis & $38(54.8)$ \\
Erythema nodosum & $11(15.7)$ \\
\hline Joint disorders & $66(94.7)$ \\
Neurologic damage & $12(18)$ \\
Ocular involvement & $27(39)$ \\
Vasculitis & $6(8.7)$
\end{tabular}

International comparison between the Erasmus MC and four other cohorts for diagnosic criteria for Behcet's disease

\begin{tabular}{|c|c|c|c|c|c|}
\hline & The Netherlands & Germany & UK & Turkey & Morocco \\
\hline $\mathrm{N}$ & 110 & 590 & 419 & 2147 & 1034 \\
\hline 8:? ratio & 1 & 1.4 & 0.5 & 1.03 & 2 \\
\hline Oral ulcers & $100 \%$ & $98 \%$ & $100 \%$ & $100 \%$ & $100 \%$ \\
\hline Genital ulcers & $79.1 \%$ & $64 \%$ & $89 \%$ & $88 \%$ & $86 \%$ \\
\hline Eryhema" nodosum & $31.8 \%$ & $42 \%$ & . & . & $16 \%$ \\
\hline Pustules* & $80.9 \%$ & $62 \%$ & . & . & $6_{4} \%$ \\
\hline Uveitis & $61.8 \%$ & $53 \%$ & $68 \%$ & $29 \%$ & $44 \%$ \\
\hline Positive pathergy test & $57.1 \% * *$ & $34 \%$ & $32 \%$ & $56 \%$ & $53 \%$ \\
\hline
\end{tabular}

CONCLUSION Behcet disease needs attention for it's multi-organ involvement. The takeover depends in severity of disease, often needing to have recourse to use: corticoids, immunosuppresive drugs, less than interferon alpha, thalidomide or anti TNF alpha. 